\title{
A VANISHING THEOREM FOR DONALDSON INVARIANTS
}

\author{
PAOLO LISCA
}

(Communicated by Ronald Stern)

\begin{abstract}
Given a smooth simply connected 4-manifold $M$, we prove that if there is a smoothly embedded 2-torus $T$ inside $M$, then the $S U(2)$-Donaldson invariants of $M$ vanish on collections of 2-homology classes, all of which are orthogonal to $[T]$ and at least two of which are multiples of $[T]$. From this we deduce obstructions to the representability of 2-homology classes of some algebraic surfaces by smoothly embedded tori, and we compute the group of self-diffeomorphisms of certain 4-manifolds with boundary.
\end{abstract}

\section{INTRODUCTION}

In this paper we prove the following vanishing theorem for Donaldson invariants, and we give some applications.

Theorem 1.1. Let $M$ be a smooth closed simply connected oriented 4-manifold with $b_{2}^{+}(M)$ odd and greater than one, and $T \hookrightarrow M$ a smoothly embedded torus with self-intersection zero. Let $k$ be an integer, $k>\frac{3}{4}\left(1+b_{2}^{+}(M)\right)$. Then the Donaldson invariants of $M$ vanish on any collection of 2-homology classes orthogonal to $[T]$ of which at least two are multiples of $[T]$.

This theorem is consistent with results announced by O'Grady [OG2]:

O'Grady obtains Theorem 1.1 for $k$ large enough when $M$ is an algebraic surface and $T$ an elliptic curve. The blow-up formula for the Donaldson polynomials [FM] together with Theorem 1.1 give a result (Corollary 3.2) already obtained by a different approach [L].

To motivate Theorem 1.2, we recall that the adjunction formula for a curve $C$ inside a complex surface $S$ is

$$
C . C+C . k_{S}=2 \text { genus }(C)-2,
$$

where $k_{S}$ is the canonical class of $S$. When $k_{S}$ is numerically effective, e.g., if $S$ is minimal irrational, this gives $C . C \leq 2 \operatorname{genus}(C)-2$. Several results which extend this inequality to smoothly embedded surfaces $\Sigma \hookrightarrow S$ have been obtained ([KM], [L], [MMR], [R]). On the other hand a naive analogue of $(*)$ for smooth emberdings is bound to fail (there are, for instance, embedded

Received by the editors May 12, 1993.

1991 Mathematics Subject Classification. Primary 57R55; Secondary 53C07, 57N13, 57R50, $81 \mathrm{E} 13$.

Key words and phrases. 4-manifolds, gauge theory, Donaldson invariants. 
spheres with self-intersection less than -2 inside a K3 surface). Nonetheless, Theorem 1.1 implies the following result, which, for the complex surfaces of the statement, is equivalent to $(*)$ for smoothly embedded surfaces of genus one:

Theorem 1.2. Let $S$ be a smooth simply connected complex surface with geometric genus greater than zero. Let $k$ be an integer, $k>\frac{3}{4}\left(1+b_{2}^{+}(S)\right)$. Suppose that the Donaldson invariant $\gamma_{k}(S)$ is a polynomial in the intersection form $q_{S}$ and the canonical class $k_{S}$, with $k_{S}^{l}$, for some $l>1$, appearing non-trivially in its expression. Then any smoothly embedded torus $T \hookrightarrow S$ with $[T] .[T]=0$ satisfies $[T] \cdot k_{S}=0$.

There are several classes of surfaces which are known to satisfy the hypotheses of Theorem 1.2, e.g., minimal smooth elliptic surfaces with geometric genus greater than two [FM] or, putting together [OG1] and [Mo], smooth surfaces $S_{n} \subseteq \mathbb{P}^{3}$ of degree $n>4$ divisible by 4 .

Observe that if $S$ is a surface satisfying the hypothesis of Theorem 1.2 and $T \hookrightarrow S$ is a smoothly embedded torus, then by Theorem 1.1 the Donaldson invariants of $S$ vanish on collections of classes orthogonal to [T] of which at least one is a multiple of $[T]$. So for such a surface a stronger version of Theorem 1.1 holds.

Theorem 1.2 can be applied to get information about the group of automorphisms of certain 4-manifolds with boundary considered in [G]. We compute the group of automorphisms of the intersection form induced by selfdiffeomorphisms for such 4-manifolds (Theorem 3.5).

\section{PROOF OF THE VANISHING THEOREM}

In order to state the result we will use to prove the vanishing theorem, we need to review briefly the results of [MMR]. Let $\left(Y, g_{Y}\right)$ be a closed, oriented, Riemannian 3-manifold, and $(X, g)$ an oriented Riemannian 4-manifold with a cylindrical end orientation-preserving isometric to $[0,+\infty) \times Y$. The results we are going to describe hold for generic metrics $g$ on $X$ exponentially decaying to the metric $g_{Y}$ along the end. We shall assume from now on to have chosen such a metric, and we shall omit it from the notation. Let $P \rightarrow X$ be a principal $S U(2)$-bundle, $\mathscr{M}^{0}(X)$ and $\mathscr{M}(X)$ respectively the based and unbased $L^{2}$-moduli spaces of ASD connections on $P$, and $\mathscr{M}_{\delta}^{0}(X)$ and $\mathscr{M}_{\delta}(X)$ the based and unbased subspaces of $\delta$-exponentially decaying $L^{2}$-connections ([Mr], [MMR], [T]).

Let $\mathscr{A}(Y)$ be the space of $S U(2)$-connections on $\eta=P \mid Y$, and $\chi(Y)$ the character variety, that is, the set of flat connections modulo gauge equivalence. Given $\Gamma \in \mathscr{A}(Y)$, denote by ad $\Gamma$ the induced connection on the adjoint bundle ad $\eta$. Recall that the set of flat connections on $\eta$ can be identified with $\mathscr{R}(Y)=\operatorname{Hom}\left(\pi_{1}(Y), S U(2)\right)$ via the holonomy representation, and $\chi(Y)$ with $\mathscr{R}(Y) / S U(2)$, where $S U(2)$ acts on the right by conjugation.

Let $t: X \rightarrow \mathbb{R}$ be any smooth function extending the projection onto the first factor over the end of $X$. There is a continuous $S O(3)$-equivariant map $\partial_{X}^{0}: \mathscr{M}^{0}(X) \rightarrow \mathscr{R}(Y)$ sending every element of $\mathscr{M}^{0}(X)$ down to the limit, for $t \rightarrow+\infty$, of its restriction to $\{t\} \times Y$, inducing a map $\partial_{X}: \mathscr{M}(X) \rightarrow \chi(Y)$. 
Given $[A] \in \mathscr{M}(X)$, let $c_{2}([A])=\frac{1}{8 \pi^{2}} \int_{X}\left|F_{A}\right|^{2} \operatorname{vol}(g)$ be the square of the $L^{2}$ norm of the curvature.

For $r \in[0,+\infty)$ and given a subset $V \subset \chi(Y)$ we shall use the notation $\mathscr{M}_{r}(X)=c_{2}^{-1}(r), \mathscr{M}(X ; V)=\partial_{X}^{-1}(V)$ and the corresponding based ones; similar notation will be used for the analogous subsets of the moduli spaces of $\delta$-decaying connections.

For $X$ equal to the complement of a smoothly embedded torus of square zero inside a smooth simply connected 4-manifold, the analysis of the ChernSimons flow has been carried out in [GM] for $S O(3)$-moduli spaces. Adapting the results of [GM] (in particular, Theorem VI.4.1) to the $S U(2)$-setup immediately yields the following:

Theorem 2.1. Let $M$ be a smooth simply connected closed 4-manifold with $b_{2}^{+}(M)$ odd and greater than one, and $T \hookrightarrow M$ a smoothly embedded 2-torus with self-intersection zero. Let $X \subset M$ be the complement of a closed tubular neighborhood of $T$ inside $M$. There exists a $\delta>0$ such that, for generic metrics on $X$ exponentially decaying to the product metric down the end, the $L^{2}$-moduli space $\mathscr{M}(X)$ is smoothly stratified with respect to the filtration

$$
\mathscr{M}(X) \supset \mathscr{M}\left(X, \chi(Y)^{t r}\right) \supset \mathscr{M}_{\delta}\left(X, \chi(Y)^{t r}\right)
$$

For any $[\omega] \in \mathscr{M}(X), \quad c_{2}([\omega]) \equiv 0 \bmod \mathbb{Z}$. Given a positive integer $k$, let $d=d(k)=4 k-\frac{3}{2}\left(1+b_{2}^{+}(M)\right)$. Then

(1) $\operatorname{dim}\left\{\mathscr{M}_{k}(X) \backslash \mathscr{M}_{k}\left(X, \chi(Y)^{t r}\right)\right\}=2 d(k)+1$,

(2) $\operatorname{dim}\left\{\mathscr{M}_{k}\left(X, \chi(Y)^{t r}\right) \backslash \mathscr{M}_{\delta, k}\left(X, \chi(Y)^{t r}\right)\right\}=2 d(k)-2$,

(3) $\operatorname{dim} \mathscr{M}_{\delta, k}\left(X, \chi(Y)^{t r}\right)=2 d(k)-6$.

Proof of Theorem 1.1. The proof is similar to those of XVI.0.9 in [MMR] and 7.1 in [L]. The condition $k>\frac{3}{4}\left(1+b_{2}^{+}(M)\right)$ assures that $\gamma_{k}(M)$ is defined. Write $M=X_{1} \bigcup_{\partial X_{1}=\partial X_{2}} X_{2}$, where $X_{1}$ is a tubular neighborhood of $T$. Let $s>0$, and let $\Sigma_{1}, \ldots, \Sigma_{d} \hookrightarrow M$ be smoothly embedded surfaces in general position, with $\Sigma_{1}, \Sigma_{2} \subset X_{1},\left[\Sigma_{1}\right]=\left[\Sigma_{2}\right]=[T] \in H_{2}(M ; \mathbb{Z})$, and $\Sigma_{i} \subset X_{2},\left[\Sigma_{i}\right]=$ $\alpha_{i-2} \in H_{2}(M ; \mathbb{Z}), i=3, \ldots, d$. We choose on the $X_{i}$ 's structures of smooth four-manifolds with cylindrical ends diffeomorphic to $[0,+\infty) \times \partial X_{i}, i=1,2$, and generic metrics $g_{1}$ and $g_{2}$ exponentially decaying to product metrics along the ends. Let $t_{i}: X_{i} \rightarrow \mathbb{R}$ be smooth functions extending the projections onto the first factor on the ends $[0,+\infty) \times \partial X_{i}$ inside $X_{i}$. Let $X_{i}^{s}=t_{i}^{-1}((-\infty, s])$ for any $s>0$, and let $M^{s}=X_{1}^{s} \amalg X_{2}^{s} / t_{1}^{-1}(s-1, s] \sim t_{2}^{-1}(s-1, s] . \quad M^{s}$ can be provided with a smooth structure diffeomorphic to $M$, and one can build generic $C^{\infty}$ metrics $g_{s}$ on $M^{s}$ interpolating $g_{1}$ and $g_{2}$. Given the surfaces $\Sigma_{i}$ there exist corresponding transverse codimension-two submanifolds $\Delta_{\Sigma_{i}}(s) \subset \mathscr{M}_{k}\left(M^{s}\right)$ so that, once an orientation for the moduli space is chosen, the value of the Donaldson invariant $\gamma_{k}\left(M^{s}\right)$ on the classes represented by the $\Sigma_{i}$ 's equals the algebraic intersection number $\sharp \bigcap_{i \geq 1} \Delta_{\Sigma_{i}}(s)$ (this does not depend on $s$, because $M^{s}$ is diffeomorphic to $M$ for all $s$ ). But we claim that for $s$ sufficiently large $\bigcap^{d} \Delta_{\Sigma_{i}}(s) \cap \mathscr{M}_{k}\left(M^{s}\right)$ is empty. By contradiction, suppose $s_{n} \rightarrow+\infty$ and $\left[A_{n}\right] \stackrel{i=1}{\in} \cap \Delta_{\Sigma_{i}}\left(s_{n}\right) \cap \mathscr{M}_{k}\left(M^{s_{n}}\right)$. Then, up to passing to a subsequence, by [MMR] there are points $x_{1}, \ldots, x_{r} \in X_{1}, y_{1}, \ldots, y_{s} \in X_{2}$ 
such that $A_{n}$ restricted to $X_{1}^{s_{n}} \backslash\left\{x_{i}\right\}$ converges modulo gauge to an $L^{2}$-ASD connection $A_{1}$ which extends to $X_{1}$ in the $C^{\infty}$ topology, up to a gauge transformation, and analogously $A_{n}$ restricted to $X_{2}^{s_{n}} \backslash\left\{y_{j}\right\}$ converges to $A_{2}$ on $X_{2}$. Moreover, there are positive integers $\left\{n_{i}\right\}$ and $\left\{m_{j}\right\}$ attached to the points $\left\{x_{i}\right\}$ and $\left\{y_{j}\right\}$ such that if $\left[A_{1}\right] \in \mathscr{M}_{e}\left(X_{1}\right)$ and $\left[A_{2}\right] \in \mathscr{M}_{f}\left(X_{2}\right)$,

$$
e+f+\sum_{i=1}^{r} n_{i}+\sum_{j=1}^{s} m_{j} \leq k=c_{2}\left(A_{n}\right) \text {. }
$$

Also, $\left[A_{1}\right]$ belongs to the intersection of analogous transverse codimension-two submanifolds $D_{\Sigma_{1}}, D_{\Sigma_{2}} \subseteq \mathscr{M}_{e}\left(X_{1}\right)$, while [ $\left.A_{2}\right]$ belongs to the intersection of submanifolds $D_{\Sigma_{3}}, \ldots, D_{\Sigma_{d}} \subseteq \mathscr{M}_{f}\left(X_{2}\right)$ in general position, which are therefore also transverse to the stratification of Theorem 2.1. If $f=k$, we have $e=r=$ $s=0$, so that $A_{1} \in \mathscr{M}_{0}\left(X_{1}\right) \cap D_{\Sigma_{1}} \cap D_{\Sigma_{2}}$. But since $\mathscr{M}_{0}=\chi\left(S^{1} \times S^{1} \times D^{2}\right)$, $\operatorname{dim} \mathscr{M}_{0}\left(X_{1}\right)=2$, and since $D_{\Sigma_{1}}$ and $D_{\Sigma_{2}}$ are in general position, this gives a contradiction. Hence we may assume $f<k$ and $e+r \geq 1$. For any given index $i \in\{3, \ldots, d\}$, if $y_{j} \notin \Sigma_{i}$ for all $j=1, \ldots, s$, then $\left[A_{2}\right] \in D_{\Sigma_{i}}$, because $D_{\Sigma_{i}}$ is closed and the sequence $\left\{A_{n}\right\}$ restricted to $X_{2}$ converges away from $\left\{y_{j}\right\}$. Hence for $i=3, \ldots, d,\left[A_{2}\right] \in D_{\Sigma_{i}}$ or $y_{j} \in \Sigma_{i}$ for some $j$, and we may choose the $\Sigma_{i}$ 's so that there are no points $y_{j}$ belonging to more than two surfaces. So by transversality of the $D_{\Sigma_{i}}$ 's we have

$$
2 d-4 \leq 4 s+\operatorname{dim} \mathscr{M}_{f}\left(X_{2}\right) .
$$

If $f=0$, then it is easy to see that $\operatorname{dim} \mathscr{M}_{f}\left(X_{2}\right)=0$, which gives $4 s \geq 2 d-4$; on the other hand $k>\frac{3}{4}\left(1+b_{2}^{+}(M)\right)$ implies $2 d>4 k$ and from $k \geq e+f+r+s$ we get $k \geq s+1$, hence $4 k \geq 4 s+4$, and we have the contradiction $4 s>4 s$. So we may assume $f \neq 0$. Since $e+f+\sum n_{i}+\sum m_{j} \leq k, f \leq k-(e+r+s)$. Therefore by Theorem $2.1 \operatorname{dim} \mathscr{M}_{f}\left(X_{2}\right) \leq 2 d+1-8(e+r+s)$, and by (*) $2 d-4 \leq 4 s+2 d+1-8(e+r+s)$, i.e., $8(e+r)+4 s \leq 5$, which gives $e+r=0$, contrary to our assumption.

\section{Applications}

As a first example of application of Theorem 1.1 we have Theorem 3.1 and Corollary 3.2, which have already been obtained ([L], Theorems 7.1 and 7.3) in the particular cases $T . T=+1$ (which imply the general cases by the blow-up formula). Indeed, Theorem 3.1 is now a special case of a more general result of Kronheimer and Mrowka [KM].

Theorem 3.1. Let $M$ be a smooth closed simply connected oriented 4-manifold with $b_{2}^{+}(M)>1$ odd, and suppose $T \hookrightarrow M$ is a smoothly embedded torus with positive self-intersection. Let $k$ be an integer, $k>\frac{3}{4}\left(1+b_{2}^{+}(M)\right)$. Let $d=d(k)=4 k-\frac{3}{2}\left(1+b_{2}^{+}(M)\right)$. Then the Donaldson invariants $\gamma_{k}(M)$ vanish on any $d(k)$-tuple of classes $\alpha_{1}, \ldots, \alpha_{d(k)} \in H_{2}(M ; \mathbb{Z})$ orthogonal to $[T]$.

Proof. Let $n=T . T>0$. The torus $T^{\prime}=T \sharp^{n} \overline{\mathbb{C P}}^{1} \subset \hat{M}=M \sharp^{n} \overline{\mathbb{C P}}^{2}$ has selfintersection zero, and by [FM] $\gamma_{k}\left(M \sharp \overline{\mathbb{C P}}^{2}\right)=\gamma_{k}(M)-2 \frac{d !}{(d-4) ! 4 !} e^{4} \gamma_{k-1}(M)+\cdots$, where $e$ is the exceptional class. Hence, if $e_{n}$ is the exceptional class of the $n$th copy of $\overline{\mathbb{C P}}^{2}$,

$$
\gamma_{k+1}(\hat{M})=\gamma_{k+1}\left(M \sharp^{n-1} \overline{\mathbb{C P}}^{2}\right)-2 \frac{d !}{(d-4) ! 4 !} e_{n}^{4} \gamma_{k}\left(M \sharp^{n-1} \overline{\mathbb{C P}}^{2}\right)+\cdots .
$$


Let $\alpha_{1}, \ldots, \alpha_{d} \in H_{2}(M ; \mathbb{Z}) \cap[T]^{\perp}$. The $\alpha_{i}$ 's, as classes in $H_{2}(\hat{M})$, are orthogonal to $\left[T^{\prime}\right]$. Hence by Theorem 1.1

$$
\gamma_{k+1}(\hat{M})\left(\left[T^{\prime}\right],\left[T^{\prime}\right],\left[T^{\prime}\right],\left[T^{\prime}\right], \alpha_{1}, \ldots, \alpha_{d}\right)=0 .
$$

On the other hand $(*)$ gives

$$
\begin{gathered}
\gamma_{k+1}(\hat{M})\left(\left[T^{\prime}\right],\left[T^{\prime}\right],\left[T^{\prime}\right],\left[T^{\prime}\right], \alpha_{1}, \ldots, \alpha_{d}\right) \\
=-2\left(e_{n} . T^{\prime}\right)^{4} \gamma_{k}(M)\left(\alpha_{1}, \ldots, \alpha_{d}\right),
\end{gathered}
$$

Since $e_{n} \cdot T^{\prime} \neq 0$, this concludes the proof.

Corollary 3.2. Let $M$ be a smooth closed simply connected oriented 4-manifold with $b_{2}^{+}(M)>1$ odd, and suppose $T \hookrightarrow M$ is a smoothly embedded torus with positive self-intersection. Let $k$ be an integer, $k>\frac{3}{4}\left(1+b_{2}^{+}(M)\right)$, and $I=\left(i_{1}, \ldots, i_{n}\right)$ a multi-index with $|I|=\sum_{j} i_{j}<d=d(k)$. Then the generalized Donaldson invariants $\gamma_{k, I}(M)$ vanish on any $(d-|I|)$-tuple of classes $\alpha_{1}, \ldots, \alpha_{d-|I|} \in H_{2}(M ; \mathbb{Z})$ orthogonal to $[T]$.

Proof. This follows from Theorem 3.1 in exactly the same way Theorem 7.3 follows from Theorem 7.1 in [L] (use the definition of $\gamma_{k, I}(M)$ and Theorem 3.1 applied to $M$ blown-up away from $T$ ).

Proof of Theorem 1.2. By hypothesis

$$
\gamma_{k}(S)= \begin{cases}a_{1} k_{S} q_{S}^{n}+a_{3} k_{S}^{3} q_{S}^{n-1}+\cdots & \text { if } p_{g} \text { is even, } \\ a_{0} q_{S}^{n}+a_{2} k_{S}^{2} q_{S}^{n-1}+\cdots & \text { if } p_{g} \text { is odd }\end{cases}
$$

with $a_{l} \neq 0$ for some $l>1$. Choose a $d$-tuple of classes $\alpha_{1}, \ldots, \alpha_{d}$ of which $l$ are equal to $[T]$ and the rest are all equal to a class $\alpha$ with $\alpha . \alpha \neq 0$. By Theorem $1.1 \gamma_{k}\left(\alpha_{1}, \ldots, \alpha_{d}\right)=0$, which implies

$$
a_{l}\left(k_{S} . T\right)^{l} q_{S}(\alpha)^{n-\left[\frac{l}{2}\right]}=0 .
$$

Remark. Theorem 1.2 can be strengthened to include the case of a torus of square zero $T \hookrightarrow \hat{S}$, where $\hat{S}$ is obtained by blowing-up a surface $S$ satisfying the hypothesis of 1.2 , and [T] is orthogonal to the exceptional classes. In fact, choosing the classes $\alpha_{1}, \ldots, \alpha_{d}$ in $H_{2}(S)$ as in the proof of Theorem 1.2, by [FM] $\gamma_{k}(\hat{S})\left(\alpha_{1}, \ldots, \alpha_{d}\right)=\gamma_{k}(S)\left(\alpha_{1}, \ldots, \alpha_{d}\right)$, and the argument works just as well.

We point out that Theorem 1.2 can be used to determine the minimal genus of smoothly embedded surfaces representing certain 2-homology classes. For example, let $S \rightarrow \mathbb{C P}^{1}$ be a smooth simply connected elliptic surface without multiple fibers with $p_{g}(S)=3$. Then there is a section $s: \mathbb{C P}^{1} \rightarrow S$, and if $C=s\left(\mathbb{C P}^{1}\right), C . C=-4$. Let $[f]$ denote the homology class of a smooth fiber of $S$. Construct a smoothly embedded surface $\Sigma \hookrightarrow S$ representing $[C]+2[f]$, using a copy of $C$ plus two smooth fibers by tubing at the intersection points. Then $\Sigma . \Sigma=0$, genus $(\Sigma)=2$, and, since the Poincaré dual of $k_{S}$ equals $2[f]$, $\Sigma . k_{S}=2$. As observed in the introduction, Theorem 1.2 applies to $S$, so [ $\left.\Sigma\right]$ cannot be represented by a smoothly embedded torus. 


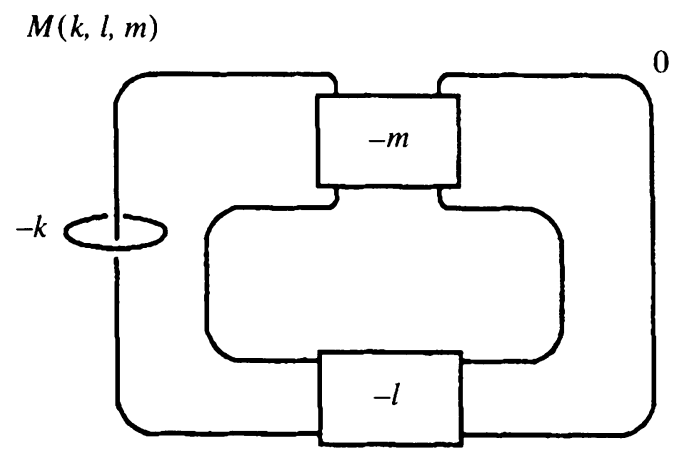

FIGURE 1

Now we give our last application. Let $k, l, m$ be positive integers. Consider the 4-manifolds $M(k, l, m)$ obtained by attaching 2-handles to $B^{4}$ along the framed links of Figure 1.

Lemma 3.4. For all $k, l, m>0$ there is a smooth embedding $i: M(k, l, m) \hookrightarrow$ $M(k, 1,1) \stackrel{l+m-2}{\sharp} \overline{\mathbb{C P}}^{2}$ such that $i_{*} H_{2}(M(k, l, m)) \subseteq H_{2}(M(k, 1,1))$.

Proof. Blow-up $l+m-2-1$ 's in a link picture for $M(k, 1,1)$ and slide them over the 0 -framed handle to get a framed link containing Figure 1 plus algebraically unlinked -1-framed unknots (see [K] for the calculus of links).

The intersection form of $M(k, l, m)$ is either isomorphic to $(1) \oplus(-1)$ or to $\left(\begin{array}{ll}0 & 1 \\ 1 & 0\end{array}\right)$, depending on $k, l, m$. It is easy to see that the group of automorphisms of either form is isomorphic to $\mathbb{Z} / 2 \mathbb{Z} \oplus \mathbb{Z} / 2 \mathbb{Z}$. Let $\mathscr{D}(k, l, m) \subseteq \mathbb{Z} / 2 \mathbb{Z} \oplus \mathbb{Z} / 2 \mathbb{Z}$ denote the image, under the natural map, of the group of self-diffeomorphisms of $M(k, l, m)$.

Theorem 3.5. If $k>3, \mathscr{D}(k, l, m) \cong \mathbb{Z} / 2 \mathbb{Z}$, generated by multiplication by -1 .

Proof. Observe first of all that there are self-diffeomorphisms of $M(k, l, m)$ which are -1 on $H_{2}$. One is visible in Figure 1, after rearranging the link in a symmetrical fashion, as a $180^{\circ}$ rotation about a vertical line in the plane of the paper. We need to prove that if $k>3$, there are no self-diffeomorphisms which are not \pm 1 on $H_{2}$. Let $V_{k}$ be a smooth simply connected minimal elliptic surface without multiple fibers with geometric genus $p_{g}=k-1 . M(k, 1,1)$ is nothing but the Gompf nucleus $N_{k} \subseteq V_{k}$ (see [G]). Hence by Lemma 3.4 $M(k, l, m)$ embeds inside $V_{k}^{l+m-2} \overline{\mathbb{C P}}^{2}$ with the image of $H_{2}(M(k, l, m))$ orthogonal to the exceptional classes. Moreover, it follows from the proof of the lemma that the image of $H_{2}(M(k, l, m))$ contains the class [ $f$ ] of a smooth fiber of the elliptic fibration on $V_{k}$. In fact, [f] comes from the class $\alpha$ given by the 2-handle attached to the 0 -framed component in the link picture. Arguing by contradiction, if there are self-diffeomorphisms of $M(k, l, m)$ which are not \pm 1 on $H_{2}$, one can generate the entire automorphism group of the intersection form and, in particular, find a diffeomorphism sending the (non-trivial) class $\alpha$ to a class $\hat{\alpha}$ different from $\pm \alpha$, which implies $\hat{\alpha} . \alpha \neq 0$. But $\alpha$ is clearly representable by a smoothly embedded torus, hence $\hat{\alpha}$ is so, and the same holds 
for their images inside $H_{2}\left(V_{k}^{l+m-2} \overline{\mathbb{C P}}^{2}\right)$. Also, by what we observed before, $\hat{\alpha}$ is orthogonal to the exceptional classes. Moreover, by [FM] Theorem 1.2 applies to $V_{k}$ if $k>3$. Hence, since the Poincare dual of the canonical class of $V_{k}^{l+m-2} \sharp \overline{\mathbb{C P}}^{2}$ equals a nonzero multiple of $[f]$ plus the Poincare duals of the exceptional classes, the remark following Theorem 1.2 implies $\hat{\alpha} . \alpha=0$, which gives a contradiction.

\section{ACKNOWLEDGMENTS}

It is a pleasure to thank John Morgan for several stimulating conversations.

\section{REFERENCES}

[D] S. K. Donaldson, Polynomial invariants for smooth 4-manifolds, Topology 29 (1990), 257-315.

[FM] R. Friedman and J. W. Morgan, Smooth four-manifolds and complex surfaces, Ergeb. Math. Grenzgeb., 3 Folge, Band 27, Springer-Verlag, Berlin and New York.

[G] R. E. Gompf, Nuclei of elliptic surfaces, Topology 30 (1991), 479-511.

[GM] R. E. Gompf and T. S. Mrowka, Irreducible four-manifolds need not be complex, Ann. of Math. (2) 138 (1993), 61-111.

[K] R. Kirby, A calculus for framed links in $S^{3}$, Invent. Math. 45 (1978), 36-56.

[KM] P. B. Kronheimer and T. S. Mrowka, Gauge theory for embedded surfaces, I and II, preprints.

[L] P. Lisca, On tori embedded in four-manifolds, J. Differential Geom. 38 (1993), 13-37.

[MMR] J. W. Morgan, T. S. Mrowka, and D. Ruberman, The $L^{2}$-moduli space and a vanishing theorem for Donaldson polynomial invariants, preprint.

[Mo] J. W. Morgan, Comparison of the Gieseker compactification and the Uhlenbeck compactifcation of moduli space, preprint.

[Mr] T. S. Mrowka, A Mayer-Vietoris Principle for Yang-Mills moduli spaces, Ph.D. thesis, Berkeley, CA.

[OG1] K. G. O'Grady, Algebro-geometric analogues of Donaldson's polynomials, Invent. Math. 107 (1992), 351.

[OG2] —_ Relations amongst Donaldson polynomials of algebraic surfaces, talk, Oberwolfach, September 1992.

[R] D. Ruberman, Smooth 2-spheres in homology $K$ 3-surfaces, Brandeis University, preprint.

[T] C. H. Taubes, $L^{2}$-moduli spaces on 4-manifolds with cylindrical ends. I, Harvard University, Cambridge.

Dipartimento di Matematica, Università degli Studi di Roma, "La Sapienza", P.le aldo MORO 2, 00185 ROME, ITALY

E-mail address: lisca@mat.uniroma1.it 